\title{
La importancia del trabajo social comunitario
}

\section{The importance of the community social work}

\author{
JOSÉ LUÍS SARASOLA SÁNCHEZ-SERRANO \\ Profesor del Departamento de Trabajo Social y Servicios Sociales de la Universidad Pablo de Olavide \\ jlsarsan@upo.es.ORCID: https://orcid.org/0000-0002-9327-0370
}

JUAN MANUEL GONZÁLEZ GONZÁLEZ

Profesor del Departamento de Trabajo Social y Servicios Sociales de la Universidad Pablo de Olavide. jgongon@upo.es

\begin{abstract}
ALICIA ROIZ VÁZQUEZ
Colaboradora interna en el Departamento de Trabajo Social y Servicios Sociales de la Universidad Pablo de Olavide. aroivaz@alu.upo.es.ORCID: https://orcid.org/0000-0002-1301-620X
\end{abstract}

Resumen: El presente artículo es resultado de una revisión bibliográfica en conjunto con una reflexión por parte de los autores. Tiene como objetivo explicar por qué es importante el Trabajo Social Comunitario. Para esta explicación, nos hemos fundamentado en un fenómeno social como es la exclusión social.

Durante la realización del artículo hemos podido observar perfectamente que todo en el campo social está relacionado y que desde la disciplina del Trabajo Social se puede trabajar para la mejora de los problemas que van surgiendo. Pero se debe de tener cuidado también con esto último: la sociedad cambian, surgen dificultades y problemáticas sociales nuevas, y es tarea del Trabajo Social y de los trabajadores sociales el ir innovando para poderles hacer frente.

En cambio, encontramos varias problemáticas sociales que a pesar de los años, las intervenciones, las perspectivas de análisis y de intervención; perduran a lo largo del tiempo. El mejor ejemplo de esto, es la exclusión social. Atendiendo a una visión naturalista podríamos decir que "siempre ha habido ricos y pobres", pero no nos cuestionamos sobre la procedencia de que siempre haya ricos y pobres.

Estudiamos la historia de la exclusión social, analizamos la situación de la población y acudimos al trabajo social para ver cómo se puede responder a esto.

Palabras clave: Trabajo Social Comunitariok, exclusión social, sociedad, comunidad, desigualdad, intervención estado de bienestar.

\begin{abstract}
This article is the result of a literature review in conjunction with a reflection on the hand that you write the same. It is intended to explain why it is important to Community Social work. For this explanation, we have informed us in a social phenomenon that is social exclusion.

During the realization of the present, is seen perfectly that everything is related and that from the discipline of Social work you can work for the improvement of the problems that arise. But we must be
\end{abstract}


careful also with this last. Society change, emerging new social problems, and difficulties, and task of Social work and social workers go to evolve tomake them front.

On the other hand, found several social problems that despite age, interventions, the prospects of analysis and intervention they endure over time. The best example of this is social exclusion. Attending a naturalistic view we could say that "there havealways been rich and poor", but we do not question about the provenance of that there is always rich and poor.

We studied the history of social exclusion, we analyzed thesituation of the population and went to social work to seehow it can respond to this.

Key words: Community Social Work, social exclusión, society, comunity, inequality, intervetion, welfare state.

\section{Introducción}

La situación actual española se encaja en un Estado de Bienestar con sistema económico capitalista, con 3.252.867 de personas desempleadas (Ministerio de Trabajo. 2017), con un saldo migratorio de 164.604 (INE, 2017). Con una esperanza de vida media con buena salud de 66,7 años (INE, 2018).

Podemos atribuir al Estado de Bienestar dos funciones principales. La primera es la intervención reglamentaria del Estado para asegurar una cierta seguridad económica a los ciudadanos a través de los sistemas de seguridad social. La segunda proveer a la población una serie de servicios y equipamientos colectivos a menor coste que en el mercado (Rodríguez, 2019).

Para el cumplimiento de dichas funciones podemos encontrar tipo de 4 políticas, en forma de intervenciones.

1. Intervenciones públicas que garantizan y protegen los derechos sociales.

2. Intervenciones públicas que influencian el nivel de ingresos.

3. Intervenciones con el objetivo de mejorar las condiciones sociales y materiales del medioambiente.

4. Intervenciones públicas para mejorar directamente las competencias de los individuos.

El Trabajo Social desde sus diferentes ámbitos (el trabajo social individual y familiar, trabajo social grupal y el trabajo social comunitario) trata de enmendar, en la medida de lo posible, los efectos que el sistema capitalista y la globalización van dejando en nuestra sociedad, como es la falta de atención integral a las personas sin hogar, el vaciamiento del trabajo comunitario apostando por un trabajo social individualizado., De ahí parte la idea de que es necesario que la población es- pañola tenga conciencia de la existencia de los trabajadores sociales y de que con ayuda de un profesional pueden ser acompañados, guiados y atendidos en sus dificultades.

A modo más concreto, el Trabajo Social Comunitario atiende a aquellas comunidades definidas y con espacio territorial delimitado. Es por ello que en este contexto el Trabajo Social se vuelve más importante, ya que surgen nuevas asociaciones y comunidades con objetivos y dificultades comunes.

Podemos atender también al trabajo social como una práctica teórica, como recoge Viscarret (2007:23-24). Lo detalla de forma que sobre entendemos el Trabajo Social como una disciplina que tiene un cuerpo teórico y podemos encontrar dos formas de la teorización de dicha disciplina.

Por un lado, encontramos que el cuerpo teórico ha ido constituyéndose de forma deductiva desde una discusión hasta un perfeccionamiento, y como en toda ciencia, éste va cambiando y renovándose con el paso del tiempo.

Por el otro lado encontramos que dicha teorización nace del punto intermedio entre la inducción y la deducción, ya que intenta de integrar estudio e investigación, con el fin de conseguir una mayor accesibilidad en la intervención profesional. Así pues, observamos como la perspectiva «práctico-teórica» es un aspecto central de la profesión.

La aplicación de esta perspectiva puede convertirse en objeto de investigación y de reflexión o bien derivar a una aplicación menos elevada y reconocida como es el conocimiento de la experiencia.

Antes de comenzar a desarrollar este artículo basado en el Trabajo Social Comunitario, creemos necesario recordar los valores que fueron definidos por la Federación Internacional de Trabajadores Sociales (FITS) en la Asamblea General celebrada en Holanda en 1972. Estos valores lo podemos resumir en la dignidad de la persona, el desarrollo personal, la capacidad de las personas para la autodeterminación y la responsabilidad civil. 
Estos fueron recogidos en el primer Código Deontológico Internacional de Trabajo Social aprobado en San Juan de Puerto Rico en 1976, que se actualizó en Australia en 2004 (FITS, https://www.ifsw.org/).

Para adentrarnos en el Trabajo Social Comunitario, es de suma importancia definir la comunidad, haremos mención a C. F. Ware (1963:7-17), quien la observa como un ambiente de interés individual para la vida individual y constituye la unidad de la vida nacional. Para la autora, la comunidad es una totalidad orgánica que crece continuamente por vida interna, es decir, todos sus integrantes participan en el crecimiento y el desarrollo de esta.

Así como es de suma importancia también conocer que como antecedentes del Estado de Bienestar tenemos tres etapas: la etapa de la caridad, la etapa de la beneficencia pública y la etapa de la asistencia social. En el Estado de Bienestar, tras la Segunda Guerra Mundial, se crea el Sistema Público de Servicios Sociales con objetivo de cubrir oficialmente las necesidades sociales, ya que sin este sistema quedarían vulnerados algunos principios fundamentales recogidos en la Constitución de 1978. Actualmente podemos dividir este sistema en dos grandes áreas. Por un lado, los Servicios Sociales Comunitarios y por el otro lado, los Servicios Sociales Especializados.

Más tardíamente, los principios del Sistema Público de Servicios Sociales que empezaron a desarrollarse desde 1982 tuvieron un gran valor como filosofía en la atención a los ciudadanos. Estos principios son entre otros: responsabilidad pública, descentralización, universalidad, globalidad, normalización, prevención... (Fernández et al., 2014:165-167).

La sociedad actual es resultado de un proceso histórico de cambios a niveles económicos, políticos, sociales, etc. Es por ello que para poder hablar de la importancia del Trabajo Social Comunitario en la actualidad debemos de prestar especial atención a los antecedentes de la Comunidad.

Lewellen (1985) divide las sociedades preindustriales en dos grandes grupos, por un lado, estarían las no centralizadas compuestas por bandas y tribus, y por otro lado estarían las centralizadas que serían las jefaturas y los estados (Malagón y Sarasola, 2006).

A modo general y haciendo alusión a la proposición de Lewellen (1985) en la clasificación de las sociedades preindustriales, podemos observar cómo se pasa de unas sociedades más nómadas, en el que las diferenciaciones sociales se dividían en la tarea que realizaba cada componente dentro del grupo, a otras sociedades más estáticas en cuanto al territorio.

Si hay algo que debamos destacar y que se da en todos los tipos de sociedades desde las tribus hasta las situadas en un sistema económico capitalista, es que todas de basan en un sistema de reciprocidad en el que se da a cambio de recibir y dentro de cada tipo de sociedad, están presentes entre sus miembros los tipos de reciprocidad.

En la alta edad media coexistieron una organización social rural con los campesinos libres y las villas en las que trabajaban esclavos con el señorío. En el medievo construyeron muchas iglesias, por lo que no podemos olvidar la importancia de las Iglesias en la comunidad, y surge el concepto de Parroquia como centro religioso que agrupaba a los habitantes de su demarcación territorial (Malagón y Sarasola, 2006).

Dando un salto a la Revolución Industrial, observamos cómo la población va creciendo en las ciudades y reduciéndose en los ámbitos rurales. La natalidad aumenta ya que las condiciones de salubridad en los barrios obreros que surgen alrededor de las fábricas son altas, así pues, las familias pobres tienden a tener más hijos porque muchos niños no sobrevivían. Así pues, la mortalidad también es alta.

Expresado generalmente, podemos decir que la exclusión social siempre ha existido, aunque sus formas hayan cambiado. Es un hecho, que los campesinos que llegaban a la nueva ciudad, los obreros y trabajadores de las minas o de las fábricas vivían marginados y en una situación de exclusión social, marcada por la fuerte división de clases y las riquezas.

Actualmente, la exclusión social no ha abandonado a nuestra sociedad. No es necesario irte a un barrio obrero para observar este fenómeno social. Con la globalización, la exclusión social es algo cada vez más notable, cuando en los grupos sociales hay personas que no visten de marca o a "la última" o simplemente no tiene la capacidad para consumir como el resto ya es marginado y excluido del grupo.

También pasa con los grupos en general, cuando unos grupos tienen la capacidad de consumir que no tiene otro con el cual se compara, acaba excluyendo a ese grupo. Esto se transforma en un problema de la comunidad, ya que dentro de esta puede haber personas y grupos en ambas situaciones, de exclusión y de "superioridad".

Estamos sumergidos en una sociedad donde el reparto de la riqueza y de los bienes es sinónimo de desigualdad, para Robert Reich (2012) la cantidad de recursos, dinero, riquezas e ingresos se acumulan en un $1 \%$ de la población. El dinero tiene la capacidad de controlar la política, así pues, los intereses económicos, la confianza en la democracia y en la economía de mercado se va deteriorando, teniendo en cuenta que los fallos de la política y la economía de influyen mutuamente dónde la desigualdad se está convirtiendo en un problema, como bien explica el Economista estadounidense. 
Cierta desigualdad es inevitable, puntualiza Reich, pero pregunta también ¿cuánta desigualdad podemos tolerar conservando una democracia y una economía que funcione para todos? Y así es como la democracia, el dinero, los recursos, los ingresos y las riquezas afectan a la comunidad y a la sociedad en general.

Para poder hablar de las riquezas a las que se refiere Reich, es inevitable pensar en la otra cara de la moneda, la pobreza. La pobreza ha sido entendida desde varios puntos en función del contexto histórico de la ciudad. Por ejemplo, en la Edad Media se entendía como una situación natural, mientras que en el desarrollo del capitalismo se define como un problema moral e individual (Gutiérrez, 2006: 23).

Debemos puntualizar en un fenómeno que es notable en nuestra sociedad desde finales del S. XX y principios del S. XXI. Es la globalización, fenómenos en el que se da la integración de varias sociedades y sus dimensiones culturales, económicas, tecnológicas y políticas. Se va generando un único mercado capitalista a nivel mundial y que tiene como características principales el libre comercio, la industrialización, unidad del sistema financiero mundial, globalización económica, aumento de las comunicaciones, aumento de los movimientos migratorios y un nuevo orden mundial.

Gracias a Torres podemos encontrar el punto de conexión entre las riquezas y pobrezas de las que habla Reich y la globalización. Existe un debate sobre la pobreza urbana de forma que, la definición de los conceptos de vulnerabilidad y exclusión van paralelos a una creciente preocupación que tiene su expresión a nivel internacional (Torres 2006:28-29)

Es necesario hacer una parada aquí para observar dónde queda el carácter comunitario. Desde las sociedades preindustriales ya mencionadas de Lewellen (Malagón y Sarasola, 2006) hasta la actualidad, podemos observar como cada vez más se han ido individualizando las acciones de los actores sociales y por consecuente se ha ido perdiendo el carácter comunitario.

Así pues, en el trabajo social empieza a primar el trabajo social individual o de caso, ya que no se hace tanta alusión al sentimiento de comunidad que había antaño y que además cada vez es más demandado. Esto último entendiendo que el trabajo social se mueve en un binomio de recurso necesidad.

En nuestra realidad, existen multitud de pueblos que van siendo abandonados o que su población se compone por un número bajo de habitantes, debido a que estos emigran a otras ciudades o metrópolis más pobladas más embriagadas de la globalización y sus ventajas como por ejemplo más empleo.

Haciendo alusión al pensamiento de Malagón (2011: 133143), creemos que efectivamente para conceptualizar la co- munidad, hay que recurrir a los conceptos de globalización y glocalización. Reconociendo así que la realidad social se ha vuelto más compleja debido a la aparición de nuevos fenómenos tales como la globalización, la globalización, la multiculturalidad, el envejecimiento, nuevos movimientos sociales...

Como repercusión de estos fenómenos se da que el Trabajo Social Comunitario debe adaptarse a cada momento con su realidad social. Buscando respuestas locales a problemas que pueden tener su comienzo en la esfera global.

Antonio López (2012:32-38) menciona unos retos para el Trabajo Social Comunitario, que nos viene como anillo al dedo para resumir la problemática social (exclusión y pérdida del carácter comunitario) que hemos ido desarrollando a lo largo de esto artículo. A grosso modo dichos retos son los siguientes.

1. Recuperar la legitimidad de la comunidad como ámbito para la acción colectiva.

2. El desapego o ruptura del vínculo social que experimentan los ciudadanos.

3. El Trabajo Social debe de ser reinventado en un entorno de crisis.

4. La capacidad de crear vínculos, de generar un movimiento conjunto para alcanzar un objetivo.

5. La adaptación del Trabajo Social a las nuevas tecnologías de la comunicación y la información.

Cuando hablamos de comunidad y sociedad en comparación podemos prestar atención al esfuerzo de Ferdinand Tönnies (Malagón y Sarasola, 2006) por hacer distinción entre ambas, al tratar de demostrar que la comunidad no es un modo deficiente de sociedad, sino de una forma cultural por derecho. Inclusive, la comunidad le parece superior.

La perspectiva que tiene Tönnies sobre la comunidad es de un organismo natural y real en el que la libertad es objetiva, orientada al pasado y las actitudes que se dan en ella son de afecto, amor, comprensión, amistad, gratitud y fidelidad. Mientras que la sociedad para él es un mecanismo artificial e ideal donde la libertad es subjetiva, orientada al progreso y al futuro, donde predominan las actitudes de egoísmo, vanidad, ambición económica y avidez de saber.

Sin olvidar que la sociedad se compone por individuos que interactúan y que los humanos somos seres sociales por naturaleza, es necesario exclamar que en la sociedad se da una estructura que, en muchos casos, genera desigualdades. Es necesario contar con recursos de integración social para atender a aquellas personas afectadas por dicha estructura, entre otras causas. 
Llegados a este punto podemos mencionar los objetivos del Trabajo Social Comunitario. De acuerdo con Fernández y López (2008: 46-53), podemos enumerar tres objetivos básicos.

En primer lugar, favorecer a una transformación estructural mediante el Estado de Bienestar; ya que toda persona está inmersa en una estructura y que la influencia de esa estructura en nosotros es inevitable.

En segundo lugar, conseguir la participación de los actores sociales para así poder llegar a producir un cambio social, mediante el desarrollo de la comunidad.

Por último, conseguir la interacción social y la identificación como seres relacionales de los actores sociales para así fomentar el trabajo en equipo, la confianza, la acción colectiva, etc. El Trabajo Social Comunitario analiza las capacidades personales, para poder fortalecer la interacción comunitaria.

Uniendo los objetivos del Trabajo Social Comunitario, los efectos de la globalización y la individualización de los actores sociales, encontramos la intervención comunitaria. De lo explicado anteriormente, parte la necesidad de promover los proyectos sociales para saldar los fallos que las estructuras reflejan en la comunidad. En suma, en el resurgimiento comunitario hallamos las economías colaborativas, que son iniciativas que tienen como objetivo fundamental fomentar el sentimiento y la participación de la comunidad orientada hacia una mejora en la cooperación comunitaria.

Un ejemplo de esta economía colaborativa es la moneda social, entre ellas podemos encontrar el Puma, en el Pumarejo (Sevilla) en el que en algunos establecimientos se permite pagar con esta moneda. Su valor es equivalente al euro, es decir, un puma es lo mismo que un euro, la diferencia es que el puma se consigue mediante un sistema de intercambio local por puntos que se anotan en una cartilla digital.

Ahora bien, estableciendo una relación directa de la exclusión social y el Trabajo Social Comunitario, podemos recoger la idea de Fernández y López (2008, p. 109-134). Esta relación se basa en que la exclusión social en todo momento debe de ser guiada por un paradigma de inclusión social, las vivencias individuales, grupales y comunitarias que viven los ciudadanos deben de tomar en consideración el actor de referencia, en este caso el Trabajo Social Comunitario.

Matos-Silveira (2015:81-91) nos explica que en los últimos años se ha ido acentuando el uso del término "exclusión». Se empezó a utilizar en los años setenta del s XX en Francia, pero no sería hasta los años ochenta cuando se empieza a utilizar con mayor intensidad en el ámbito de las políticas públicas y de las propias Ciencias Sociales europeas. La autora nos muestra seis desafíos del desarrollo profesional ante los procesos de exclusión/inclusión. En estos desafíos recoge quejas, recelos, advertencias, conflictos éticos, etc. Que recogió de la realización de grupos focales y entrevistas en profundidad a veinticuatro trabajadores/as sociales.

1. Relacionado con las presiones del sistema burocrático institucional.

2. Relacionado con el ciclo vicioso de intercambio de favores profesionales dada la carencia de protocolos efectivos de coordinación institucional.

3. Relacionado con la falta de recursos intermedios socio-sanitarios para la población en situaciones de extrema exclusión.

4. Relacionado con el desajuste existente entre el tiempo institucional y el tiempo profesional.

5. Relacionado con la incomunicación entre los profesionales.

6. Relacionado con el significado social de la profesión.

Recordando la información anteriormente detallada sobre la situación actual, estamos en una sociedad de las redes y la información. Con estas redes sociales se van creando nuevas comunidades cibernéticas, y este es un aspecto que también ha detenerse en cuenta; porque en ellas también se dan conflictos y necesidades. De tal forma que a los retos propuestos por Matos-Silveira, le añadiríamos otro nuevo: "Relacionado con las nuevas tecnologías y las nuevas generaciones".

No podemos dejar atrás que el Trabajo Social Comunitario se encarga de estudiar con un carácter exploratorio la comunidad, hacer un diagnóstico social con carácter reflexivo, realizar una planificación con carácter teórico, ejecutar el Plan (carácter práctico), posteriormente evalúa (de nuevo carácter reflexivo) y por último sistematiza los conocimientos a través de la elaboración de teorías (Malagón y Sarasola, 2006).

Si sumamos la necesidad de los proyectos sociales con la tarea del Trabajo Social Comunitario, podremos obtener el instrumento para arreglar la mayoría de los desajustes existentes en la comunidad. Pero para ello el trabajo social comunitario debe promover la dinamización del proceso de acción colectiva, así como promover la constitución de un grupo, animar al grupo, asistirle en la construcción de un diagnóstico comunitario y en la elaboración de un proyecto comunitario, acompañarlo en la acción, etc. (Barbero y Cortés, 2005:17-24).

A lo largo de la historia del Trabajo Social, el profesional ha ido encontrándose con desafío ante situaciones de exclusión. Es necesario hacer un paréntesis y recordar que han 
ido apareciendo nuevos significados a los problemas sociales como por ejemplo pobreza, cuestión obrera, exclusión social. Son términos que dependiendo del contexto sociopolítico donde se emplean y los valores predominantes de la época, van cambiando de sentido y significado (Matos-Silviera, 2015:81-91).

En este momento es cuando se hace necesario conocer la función principal de los Servicios Sociales.

"Los Servicios Sociales Especializados o Comunitarios, se dirigen a sectores concretos de la Población, y están destinados a la atención específica de colectivos y ciudadanos ante situaciones de grave necesidad, ofreciendo respuesta escenarios de especial complejidad". (Fernández, T. et al., 2004:165).

Las funciones a grosso modo de un trabajador social comunitario son: facilitador de procesos en la comunidad, comunicador, organizador, observador, incitador, mediador, asesor, informador, defensor, formalizador, catalizador, animador y otras que estarán por venir.

Pero, profundizando más en las funciones del trabajador social comunitario, y a sabiendas que los centros de servicios sociales comunitarios se conforman por 5 especialidades, vamos a nombrar alguna de las funciones del trabajador social comunitario en dichas especialidades.

- Servicio de Información, Valoración y orientación (SIVO): donde el trabajador social se encarga de informar, valorar, orientar y asesorar.

- Servicio de Ayuda a Domicilio (SAD): donde el trabajador social debe formar a los auxiliadores que van a intervenir además de seleccionar las técnicas de intervención y la supervisión de estos auxiliadores.

- Servicio de Cooperación Social (COSO): aquí se debe de formar e informar a los voluntarios, fomentar las campañas de voluntariado, garantizar que se cumplan sus derechos.

- Servicio de Convivencia y Reinserción Social (CORE): en este ámbito el trabajador social comunitario debe fomentar las posibilidades de mejora en las condiciones personales y sociales para la convivencia, tienen que tener carácter preventivo y en suma deben ofrecer alternativas/apoyo a aquellas personas con dificultades para la convivencia y/o sociabilidad.

Por un lado, para garantizar que estas prestaciones básicas pertenecientes, recordemos, a un Sistema Público de Servicios Sociales, salió la Ley de Base de Régimen Local. Esta establece que en cada población con más de 20.000 hab- itantes debía de existir un Centro de Servicios Sociales, las que tenían un número de habitantes inferior, tendrían este Centro de forma mancomunal.

Por otro lado, para garantizar una buena atención social, la intervención comunitaria se desarrolla generalmente en niveles (Malagón y Sarasola, 2006).

1. Estudio de la comunidad donde se realiza una delimitación espacial del territorio en el que se pretende intervenir, una descripción de la comunidad, un análisis de la población, un estudio de las redes sociales y familiares, así como la existencia de Sociedades, asociaciones e instituciones.

2. Organización de la comunidad donde podemos encontrar una organización interna y una externa de la misma. En la primera se da que la organización proviene de la propia comunidad, en la que cualquier miembro de la comunidad puede organizar una reunión con una asociación de la misma comunidad. En la segunda se da cuando la organización proviene de personas ajenas a esa propia comunidad como por ejemplo un trabajador social ofrece información a las asociaciones de una misma comunidad para que estas se reúnan.

3. Desarrollo comunitario, este nivel se da como respuesta a los efectos de la II Guerra Mundial, enfocado a un desarrollo de base económica y dirigido a países y zonas con condiciones sociales y económicas muy deterioradas.

No podemos olvidar a la hora de estudiar una comunidad, atender a diversos aspectos que nos recuerda Caroline y Ware (1963:7-17), como son la situación y organización geográfi$\mathrm{ca}$, los antecedentes históricos que conforman el patrimonio de la comunidad, el proceso de cambio cultural al que está sometida constantemente, las características de la población de la misma, la organización social, las condiciones económicas, la organización religiosa, las características políticas, la familia, los tipos de personalidad de los individuos que se desarrollan en ella, las actitudes y valores de sus miembros y los problemas que se dan dentro de esa comunidad.

De acuerdo con Acebes y Delgado, "el Trabajo Social Comunitario se trata de un saber hacer válido para profundizar en los mecanismos relaciones entre las personas, que implique un enriquecimiento de los mismos y a su vez un reforzamiento de la participación" (2012:105).

En la actualidad, todos aquellos que se dedican o nos queremos dedicar a lo que la profesión de Trabajo Social im- 
plica, necesitamos reflexionar sobre todo lo que constantemente pasa a nuestro alrededor.

Y para finalizar este artículo, nos gustaría hacerlo mencionando la aportación que realiza Rodríguez (2017:57-59) desde el Trabajo Social Comunitario haciendo alusión a los derechos humanos, los valores y los dilemas éticos. Exactamente cuando dice "Los principios éticos constituyen la base de la defensa de los valores para los trabajadores sociales, frente a los retos que presentan sus contextos de intervención".

Como bien explica la autora, el trabajo social es el resultado de la interacción que se da entre individuo y sociedad, y es evidente que esta profesión debe fundamentarse en unos valores y en una ética para cumplir los objetivos en condiciones de calidad.

En esta profesión debemos de llevar los derechos humanos por delante y por encima, ya que, velando por el cumplimiento de estos, podremos conseguir el bienestar de las personas.

\section{BIBLIOGRAFÍA}

Acebes, R. , Delgado, L. (2012). Modelos teóricos y campos de intervención en el ámbito comunitario. Reflexiones sobre una experiencia de Trabajo Social Comunitario en España. Conclusiones. En Del Fresno, M., Segado, S. \& López, A.,. Trabajo Social con Comunidades en el Siglo XXI. Madrid .Universitas.

Barbero, J. M. , Cortés, F. (2005). Trabajo Social Comunitario, organización y desarrollo social. Madrid: Alianza Editorial.

Fernández, T. , Ponce de León ,L y Andrés ,S. (2014). Historia del Trabajo Social en España. En Fernández, T. , De Lorenzo, R. . Trabajo Social, una historial global. Madrid: Mc Graw Hill Education.

Fernández, T. , López, A. (2008). Trabajo Social Comunitario: afrontando juntos los desafíos del siglo XXI. . Madrid: Alianza Editorial.

Federación Internacional de Trabajadores Sociales. Recuperado el 5 de noviembre de 2018 de https://www.cgtrabajosocial. es/fits federacion internacional trabajadores sociales.

INE (Instituto Nacional de Estadística). (2017). Movimientos migratorios exteriores. Estadística de migraciones. Recuperado el 12 de noviembre de 2019 dehttp:// www.ine.es/dyngs/INEbase/es/operacion.htm?c=Esta- distica C\&cid $=1254736177000 \&$ menu=ultiDatos\&i$\mathrm{dp}=1254735573002$.

Lewellen, Ted C. (1985). Introducción a la antropología política. En Malagón, J.L. y Sarasola, J.L. (2006). Fundamentos del Trabajo Social Comunitario. Aconcagua Libros. Textos Universitarios.

López, A. (2013). Altruismo, cooperación, ciudadanía: fundamentos del Trabajo Social Comunitario.. En Del Fresno, M., Segado, S. y López, A .Trabajo Social con Comunidades en el Siglo XXI. Madrid .Universitas.

Malagón, J.L. , Sarasola, J.L. (2006). Fundamentos del Trabajo Social Comunitario. Aconcagua Libros. Textos Universitarios.

Malagón, J.C. , Malagón, J.L. (2011). "Trabajo Social Comunitario". En Cordero., Cordero N., y Fernández, M.I. El Mosaico de la Intervención Social, métodos y conceptos en Trabajo Social. Sevilla: Aconcagua.

Matos-Silveira, R. (2015). "Desafíos del desarrollo profesional del trabajo social ante situaciones de exclusión". En Cruz, B., Bembibre,J. y Barranco , J. Herramientas para la reflexión de la práctica de la intervención social. Granada: El genio Maligno.

Ministerio de Trabajo. Gobierno de España. Datos de Interés. Paro registrado. Recuperado el 12 de noviembre de 2018 de http://www.mitramiss.gob.es/.

Reich, R. (2012). Inequality for all. [Documental]. Recuperado el 12 de noviembre de 2018 de https://youtu.be/x4Ny6OImOGE.

Rodríguez Álvarez, M. D. (2017). Familia. Revista de Ciencias y Orientación Familiar. N. ${ }^{\circ}$ 55. P. 57-69. Derechos humanos, valores y dilemas éticos: aportaciones desde el trabajo social comunitario. Recuperado el 5 de noviembre de 2018 de https://dialnet.unirioja.es/servlet/articulo?codigo $=6151245$.

Rodríguez, M. J. Estado del Bienestar en la sociedades avanzadas. [PPT].Trabajo no publicado. Estado y Sociedad de Bienestar. Universidad Pablo de Olavide. Sevilla. 6 enero 2019.

Torres, F.J. (2006). El análisis territorial aplicado al estudio de zonas urbanas marginadas, el caso de Polígono Sur en Sevilla. P. 23 \& 28-29.

Viscarret, J.J. (2007). Modelos y métodos de intervención en Trabajo Social. Madrid :Alianza Editorial.

Ware, C. (1963). Estudio de la Comunidad. Buenos Aires :Humanitas. 